\title{
Recent Results from Milagro and Prospects for HAWC
}

\author{
John Pretz * \\ Los Alamos National Lab \\ E-mail: jpretz@lanl.gov \\ Stefan Westerhoff *广 \\ University of Wisconson-Madison \\ E-mail: westerhoffephysics.wisc.edu
}

The High Altitude Water Cherenkov (HAWC) observatory is a new experiment for observing 50 $\mathrm{GeV}$ to $100 \mathrm{TeV}$ photons from high energy gamma ray sources. The experiment is under construction at Sierra Negra, Mexico and will be comprised of 300 large water tanks instrumenting an area of 150x150 meters. HAWC is the next generation of the Milagro experiment which measured multi-TeV emission from the Galactic plane resolving sources and measuring diffuse emission. HAWC will feature approximately 15 times the sensitivity of the Milagro experiment and will be used to measure and constrain particle acceleration in the Galaxy.

35th International Conference of High Energy Physics - ICHEP2010,

July 22-28, 2010

Paris France

\footnotetext{
${ }^{*}$ For the Milagro and HAWC Collaborations

$\dagger$ Speaker.
} 


\section{Introduction}

The High Altitude Water Cherenkov (HAWC) observatory is an experiment for measuring high energy gamma rays from Galactic and extragalactic sources. The experiment consists of 300 Cherenkov detectors made with large water tanks instrumented with photo-multiplier tubes (PMTs). These tanks detect Cherenkov light from charged particles reaching the ground when a high energy gamma ray interacts in the atmosphere above the experiment. The experiment is sensitive to gamma rays of energy between $50 \mathrm{GeV}$ to $100 \mathrm{TeV}$ and will continuously observe the entire overhead sky. The experiment is under construction at Sierra Negra in Mexico and is anticipated to begin science operations in 2012 and complete construction in 2014.

HAWC is the successor to the Milagro experiment [1][2][3][4] which operated in the Jemez mountains above Los Alamos, New Mexico from 2000 to 2008. Milagro made important contributions to the understanding of Galactic sources of high energy emission and to the acceleration and propagation of particles in the Galaxy by discovering new multi-TeV emission including diffuse emission from the Galactic Plane, diffuse emission from the Cygnus region of the Galaxy and emission coincident with many Fermi sources. The Milagro observations hint at what the scientific return of HAWC will be toward understanding the acceleration and propagation of cosmic rays in the Galaxy.

Section 2 describes the design of the HAWC presents the anticipated performance. Section 3 contains a summary of the Milagro results and discusses the scientific return that HAWC will bring.

\section{HAWC Experiment and Anticipated Performance}

The HAWC experiment will consist of 300 water tanks each 7.3 meters in diameter and 5 meters high. The tanks are commercial steel water tanks with an opaque sealed bladder to contain the water. The tanks will be arranged over an area of approximately 150x150 meters. Each tank will contain three 20 centimeter PMTs re-used from the Milagro for a total of 900 channels. The PMTs will be positioned at the bottom of the water tanks. Because the PMTs observe Cherenkov light from mostly down-going air showers, the PMTs observe the water roughly in a cone above the PMT with an opening angle of some 40 degrees, the Cherenkov angle of water. The depth of the PMTs is chosen to provide coverage of the entire 150x150 meter area with the available PMTs.

The experiment is being constructed at 4100 meters elevation at the Sierra Negra mountain in Mexico at a latitude of 18.99 degrees. The Large Millimeter Telescope is near to the HAWC site and HAWC will re-use existing infrastructure for power, internet, and transportation.

The front end electronics for the experiment will be re-used from Milagro. The signals from the PMTs will be run to a central counting house and passed through custom electronics which pick off and shape the PMT signal and provide a square pulse indicating when the the PMT signal crosses pre-defined photo-electron thresholds. The time that a PMT signal spends above threshold is used to determine the amount of charge that a PMT detected. The profile of the charge of an event in the plane of the array is fit to a Gaussian distribution in order to identify the shower core - the location the original particle would have struck the ground. The time that PMT signals were 


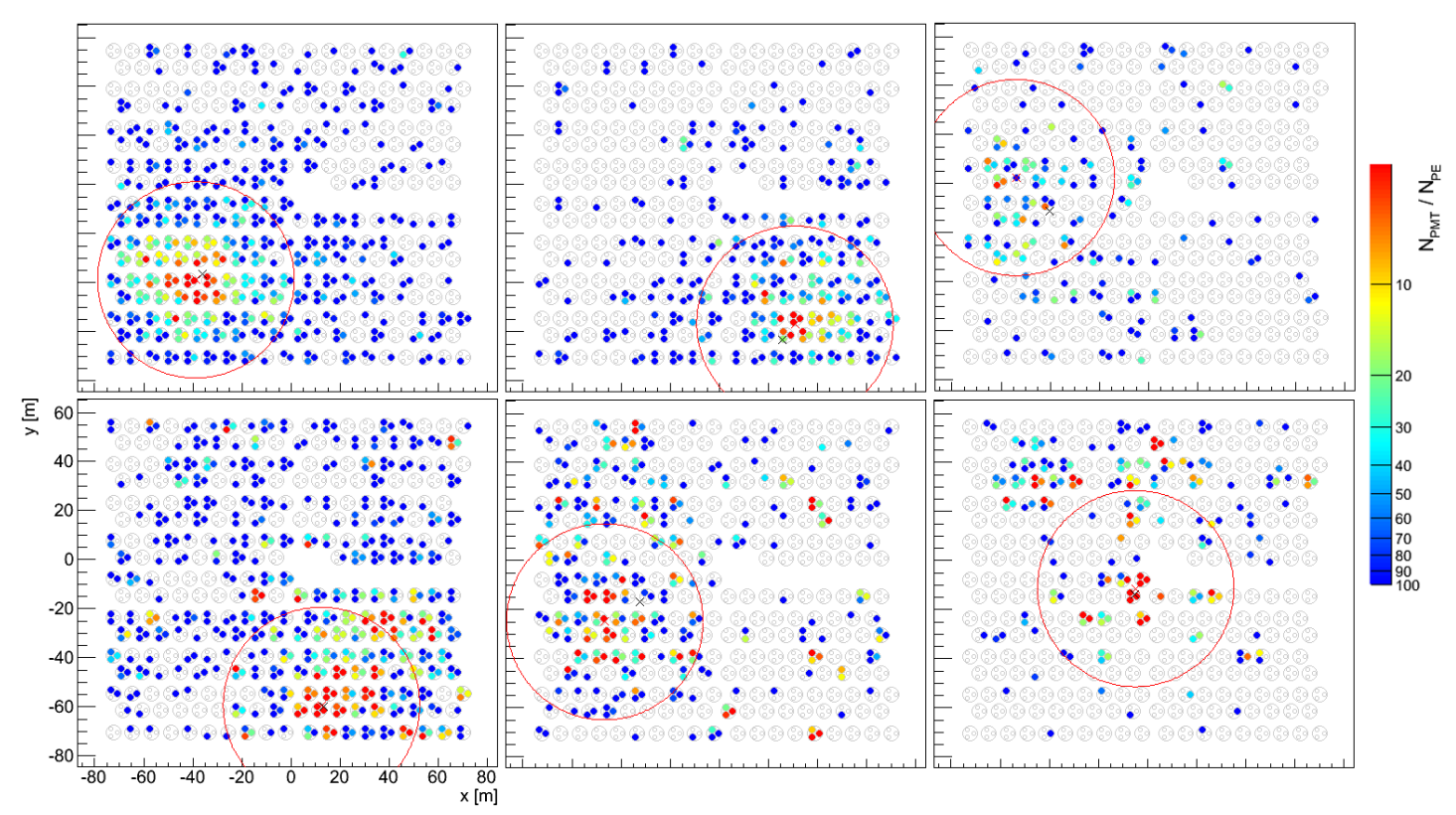

Figure 1: Simulated gamma ray and cosmic ray events. The top three panels show simulated gamma ray and the bottom three show simulated cosmic ray events. Shown are the location of HAWC PMTs and how much charge each channel received. A 40 meter circle has been drawn over the core of the air shower. The color scale is chosen to be the number of hit PMTs in the event divided by the number of PEs in each channel. Red PMTs are hit harder. Note the localized depositions of energy away from the shower core for the hadronic events.

detected is used to determine the direction of the incident air shower. Depending on the number of PMTs observing light, events can be reconstructed with an accuracy between 1.0 and 0.1 degrees.

The layout of the PMTs in HAWC provides for the ability to distinguish gamma ray events from the larger cosmic ray background. The high rate of cosmic-ray induced air showers is the chief difficulty in identifying sources of gamma ray events. The water-Cherenkov technique for gamma ray detection relies on the presence of penetrating particles (mostly muons) characteristic of a hadronic cascade in order to identify cosmic ray events.

An electromagnetic shower initiate by a gamma ray has a very smooth distribution of shower particles - positrons, electrons and gamma rays - which produce visible light in the first meter of water when striking a tank. Hadronic showers from cosmic rays are clumpy in the distribution of particles and have many muons in them. The muons in a hadronic air shower penetrate through the water tank and pass close to the PMTs resulting in characteristically larger signals at the location where muon energy deposition occurs.

Figure 1 shows some simulated gamma ray and cosmic ray events in the experiment. The number of photo-electron (PEs) detected in a channel is displayed after being scaled by the total number of hits in the detector. The reconstructed core of the shower is also displayed. The shower core contains the greatest concentration of high energy particles and it is difficult to discern penetrating particles in the shower core. For this reason, we exclude a 40-meter circle around the core and search for localized regions of high-energy deposition outside of that circle. Note that the gamma rays have no such deposition and the hadron events do. 
The HAWC design differs from the Milagro design in several important ways. First, the altitude of HAWC is 4100 meters where Milagro was just over 2600 meters. Since the increased altitude places the experiment closer to the peak of the air shower development, HAWC will be able to detect showers of lower energy than Milagro as well as providing more information for the showers that are observed. This results in improved energy threshold, energy resolution and angular resolution for HAWC over Milagro. Additionally, the PMT layout allows for identification of penetrating particles across the entire area of the detector. The Milagro experiment was formed by a central 80x60 meter pond and and an array of small water tanks over an area of 200x200 meters. Though the core of a shower in Milagro could be reliably reconstructed over the entire 200x200 meter footprint of the detector, only the pmts at the 30x50 meter bottom of the water pond could be used for identifying hadronic events. This factor of 15 improvement of useful area for gamma / hadron separation gives large improvement in overall sensitivity.

Panels a, b and c of Figure 2 summarize the improvements of HAWC over Milagro. HAWC will have significantly more effective area than Milagro at lower energies due primarily to the higher altitude. HAWC will be much better at distinguishing gamma ray air showers from cosmic ray air showers at all energies due to the much larger area of deep water for finding muons. These improvements combined with the nearly 4 times improvement in the angular resolution gives HAWC an overall anticipated factor of 15 improvement in sensitivity over Milagro. It should be noted that these improvements are based on direct application of the Milagro algorithms on HAWC simulation. The flattening of angular resolution at high energies, for instance, may improve with algorithms more optimized for HAWC.

Panel d of Figure 2 exhibits the sensitivity of HAWC in the context of other high energy gamma ray experiments [5][6]. Direct comparison to the Imaging Atmospheric Cherenkov Telescopes (IACTs) is complicated by the fact that IACTs are pointed at a single source and have approximately one degree field of view whereas HAWC will continuously observe the entire overhead sky. The comparison is done by assuming that the IACTs observe a source for 50 hours. HAWC is unique among high-energy gamma ray experiments because of its sensitivity at high energies, sensitivity to sources extended by more than a degree, and ability to continuously monitor the sky.

\section{Milagro Results and Discussion}

Milagro made important contributions to the understanding of high energy sources and cosmic ray production and propagation in the Galaxy and the results hint at what the scientific return of HAWC will be. Figure 3 shows the results of the Milagro survey of the Galactic plane. Depending on the source details, the median energy of gamma rays contributing to this map are between 10 and $50 \mathrm{TeV}$. In this map, 6 sources are identified at more than 5 standard deviations after accounting for random trials: The Crab, MGRO J2019+37, MGRO 1908+06, MGRO 2031+47 as well as 0FGL J2229.0+6114 and 0FGL J0634.0+1745 from the Fermi Bright Source List. In all cases, the sources are coincident with bright pulsars observed by Fermi. Emission is also seen coincident with other sources from the Fermi Bright Source List with lower significance. Even after subtracting the known emission, diffuse emission at $20 \mathrm{TeV}$ was observed from the Galactic Plane in Milagro data, most notably in the Cygnus region of the Galaxy. This emission exceeds the estimate made 

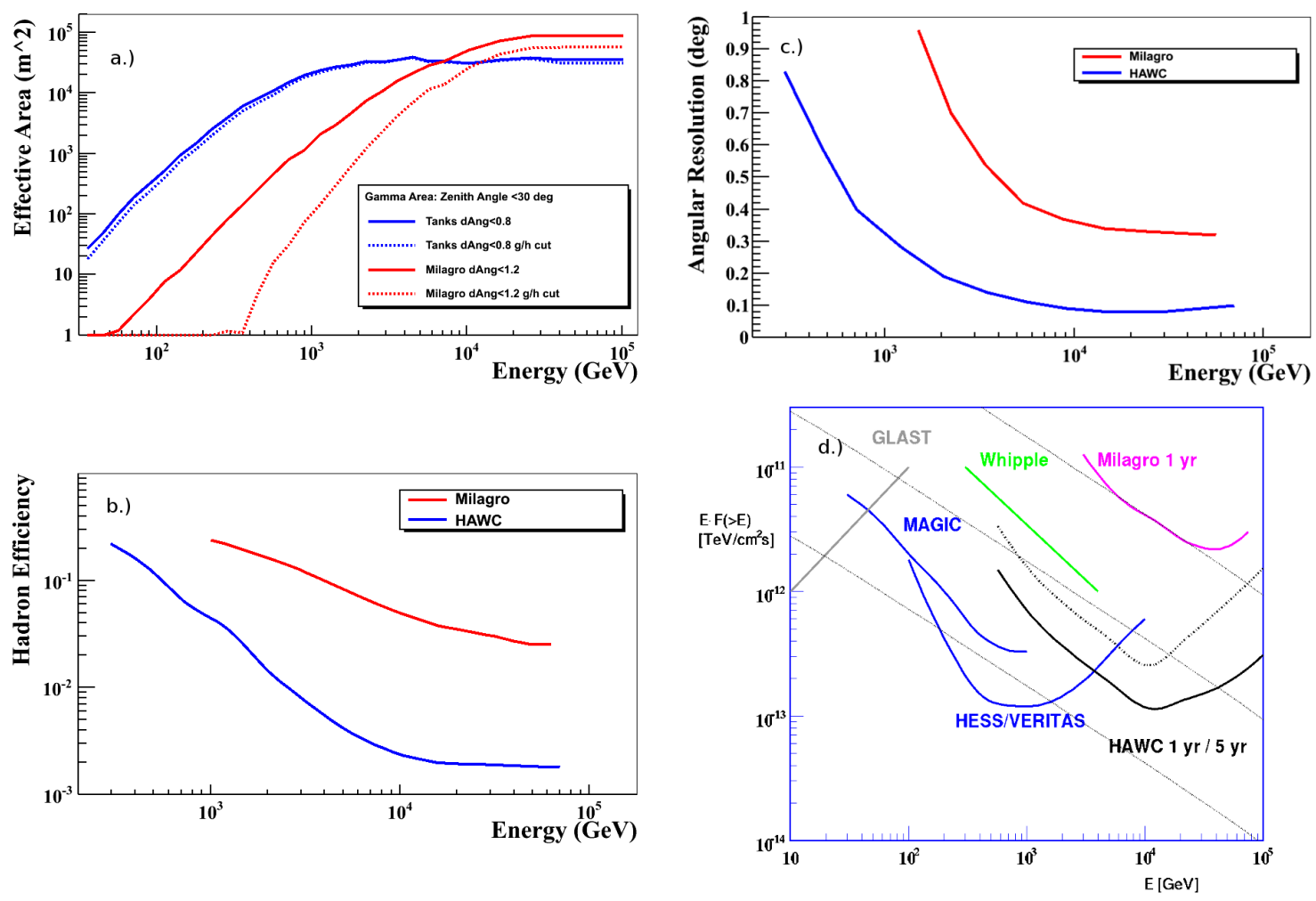

Figure 2: Illustration showing the sensitivity of HAWC and Milagro as a function of primary gamma-ray energy. Panel (a) shows the effective area for HAWC and Milagro. HAWC and Milagro have similar effective area at high energies, but HAWC has a $4 \mathrm{x}$ lower threshold, principally due to the higher elevation. This results in a 2 order of magnitude increase in the effective area below $1 \mathrm{TeV}$. Panel (b) shows the efficiency of the gamma hadroncut (described in the text) for passing hadronic background when the efficiency for accepting gamma-ray events is fixed at $50 \%$.HAWC will reject approximately $10 \mathrm{x}$ more background than Milagro at similar energies. Panel (c) shows the angular resolution of Milagro and HAWC vs energy. Panel (d) shows the sensitivity of HAWC as compared to other gamma ray observatories for 1 year (dotted line) and 5 year (solid line) observations of a source with a Crab-like spectrum.

by the GALPROP model of Galactic diffuse emission by between 4 and 8 times depending on the region.

The origin of the excess in the diffuse emission is unclear. Furthermore, it is not known how much of the diffuse emission is truly diffuse and how much is due to undetected discrete sources. Certainly the low-significance coincidences between Milagro data and Fermi sources suggest that there are a number of sources just below the detection threshold of Milagro. It may be that the diffuse excess is due to cosmic ray interactions near acceleration sites while cosmic rays are still 'young' and have not yet lost energy to propagation in the Galaxy. Such interaction has been seen by HESS [7], for instance, in the Galactic ridge. In such a case, HAWC would see an overall reduction of the diffuse emission as more discrete sources are identified. In any case, the HAWC measurements will outline cosmic ray interaction in the Galaxy and help inform whether they are interacting preferentially near sources or in the bulk Galaxy. 


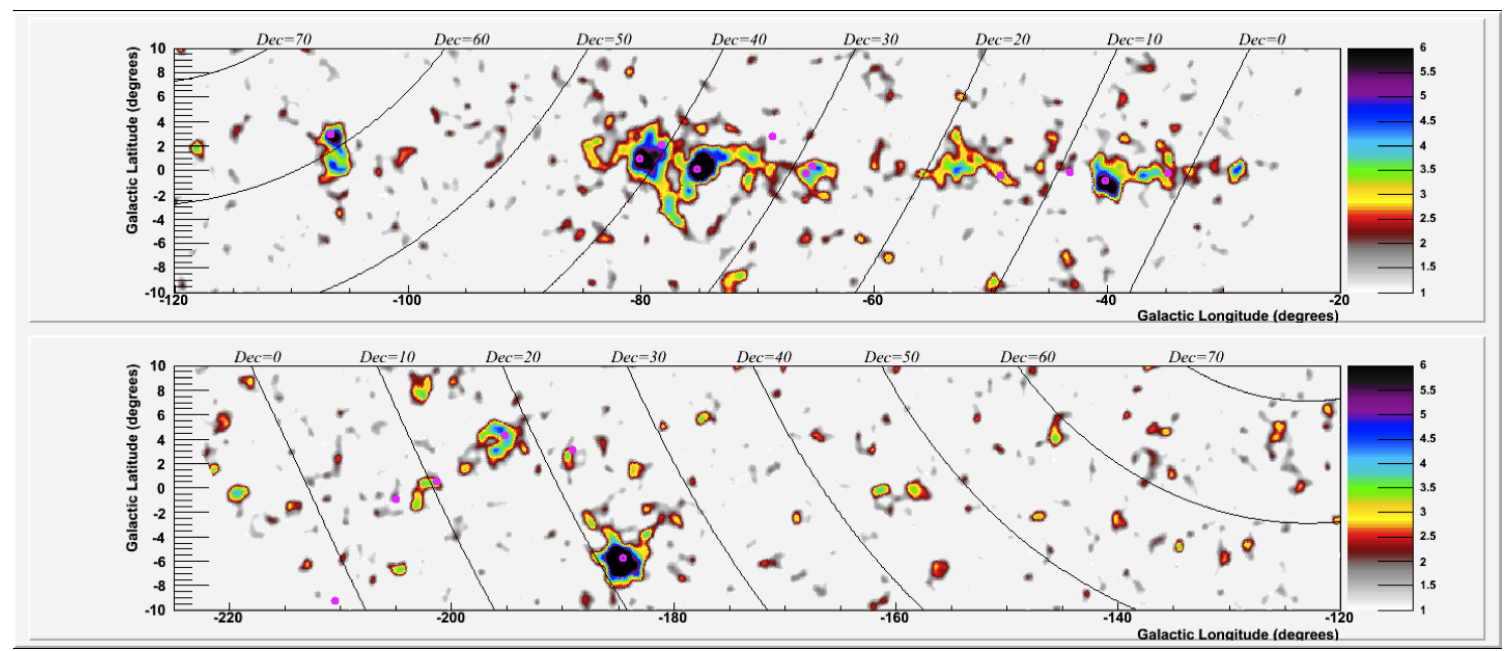

Figure 3: The Milagro sky survey in Galactic coordinates. The color scale indicates the statistical significance of each point. Objects from the Fermi Bright Source List have been overlaid in pink and the associations are apparent. The Cygnus region is evident at a Galactic longitude of 70 degrees.

\section{Conclusions}

The HAWC experiment is a next-generation multi- $\mathrm{TeV}$ gamma ray observatory currently under construction. Through a combination of a high altitude site and re-deployment of the Milagro PMTs, HAWC will achieve superior ability to distinguish gamma ray primaries from hadronic primaries and better angular resolution giving an approximately 15 -fold sensitivity increase to typical Galactic sources. With its improved sensitivity, HAWC will resolve many more multi-TeV sources, and better estimate how much of the emission in the Galaxy is truly diffuse and how much is confined to sources illuminating cosmic ray acceleration and propagation in the Galaxy.

\section{References}

[1] A.A. Abdo et al, Discovery of TeV Gamma-Ray Emission from the Cygnus Region of the Galaxy, ApJL 658 (2007) L31-36.

[2] A.A. Abdo et al, TeV Gamma-Ray Sources from a Survey of the Galactic Plane with Milagro, ApJL 664 (2007) L91-94.

[3] A.A. Abdo et al, A Measurement of the Spatial Distribution of Diffuse TeV Gamma Ray Emission from the Galactic Plane with Milagro, ApJ 688 (2008) 1078-1083.

[4] A.A. Abdo et al, Milagro Observations of Multi-TeV Emission from Galactic Sources in the Fermi Bright Source List, ApJL 700 (2009) L127-131.

[5] F. Aharonian et al, Observations of the Crab Nebula with H.E.S.S, A\&A 457 (2006) 899-915.

[6] V. A. Acciari et al, The Discovery of Gamma-Ray Emission from the Blazar RGB J0710+591, ApJL 715 (2010) L49-55.

[7] F.Aharonian et al, Discovery of Very-High-Energy Gamma-Rays from the Galactic Centre Ridge, Nature 439 (2006) 695-698. 